\title{
Tecnura
}

\section{Análisis de la intervención antrópica en cuerpos de agua: caso caño Banderas, en el municipio Puerto López (Meta, Colombia)}

\section{Analysis of anthropic intervention in water bodies: caño Banderas case in the Puerto Lopez (Meta, Colombia) Municipality}

\author{
Hernando Castro Garzón ${ }^{(0)}$, Dagoberto Torres Flórez ${ }^{2}$, Nadeska Jenniffer Gallardo Lichaa $^{(+6}$
}

Fecha de recepción: 23 de Diciembre de 2019

Fecha de aceptación: 6 de Mayo de 2020

Cómo citar: Castro-Garzón., H. Torres-Flórez., D. y Gallardo-Lichaa., N.J. (2020). Análisis de la intervención antrópica en cuerpos de agua: caso caño Banderas, en el municipio Puerto López (Meta, Colombia). Tecnura, 24(65) 77-84. DOI: 10.14483/22487638.15772

\section{Resumen}

Contexto: Puerto López se ubica en el nororiente del departamento del Meta, una distancia de $90 \mathrm{ki}-$ lómetros de la capital, Villavicencio; es conocido como el centro geográfico de Colombia, y se caracteriza por ser una zona de riqueza hídrica. El caño Banderas es parte de la cuenca del río Meta, al que efluyen el $33 \%$ de las aguas negras del casco urbano, y que se encuentra contaminado por las aguas residuales y basuras.

Método: A través de un enfoque mixto se recopiló información por medio de entrevistas semiestructuradas y encuestas, procesadas en Atlas.ti 7 y SPSS 20 , respectivamente, estableciendo relaciones para la interpretación de resultados.

Resultados: Se estableció que los impactos generados no han sido mitigados a pesar de los diferentes programas determinados en la planeación municipal; la desigualdad en la condición ciudadana permite la ocupación como el detrimento de zonas de protección ambiental, a la vez que facilita los vertimientos y el depósito de residuos sólidos en ellas.
Conclusiones: Los análisis de la intervención antrópica de cuerpos de agua permiten establecer responsabilidades en los impactos negativos de la zona, así como pormenorizar acciones de mejora para su recuperación acordes al territorio.

Palabras clave: afluentes, comunidad, contaminación, sostenibilidad.

Abstrac

Context: Puerto López is located in the northeast of the Department of Meta, is known as the geographic center of Colombia, is located at a distance of ninety kilometers from the capital, Villavicencio; It is characterized as an area of water wealth. The Banderas pipe is part of the Meta River Basin to which $33 \%$ of the urban sewage effluents effluent, establishing that it is contaminated by sewage and garbage.

Method: Through a mixed approach, information was collected through semi-structured interviews and surveys, processed in Atlas.ti 7 and SPSS 20 respectively, establishing relationships for the interpretation of results.

1 Profesional en Administración de Empresas, especialista en Ecología y Medio Ambiente, máster en Gestión Ambiental y Sostenible, PhD en Desarrollo Sostenible. Docente Universidad de los Llanos, Villavicencio, Meta, Colombia. hcastro@unillanos.edu.co

2 Profesional en Administración Pública, magíster en Administración de Empresas con énfasis en administración de proyectos, doctorando en Ciencias Económicas y Administrativas. Docente, Universidad de los Llanos, Villavicencio, Meta. Colombia. dtorres@unillanos.edu.co

3 Profesional en Administración, Maestría en Administración de Empresas, PhD en Ciencias Gerenciales. Docente, Universidad de Sabaneta, Sabaneta, Antioquia. Colombia. nadeska.gallardo.@gmail.com 
Results: It was established that the impacts generated have not been mitigated despite the different programs determined in municipal planning; The inequality in the citizen status allows the occupation as the detriment of environmental protection zones as well as facilitates the dumping and deposit of solid waste in them.
Conclusions: The analysis of the anthropic intervention of water bodies allows to establish responsibilities in the negative impacts of the area as well as to detail improvement actions for their recovery according to the territory.

Keywords: tributaries, community, pollution, sustainability.

\section{INTRODUCCIÓN}

La contaminación del agua es la alteración de sus características naturales, principalmente producida por la actividad humana, que la hace total o parcialmente inadecuada para el consumo o como soporte vital de plantas y animales (Peñaloza Paez, 2012) los problemas por contaminación se incrementan cada día (Barba Ho, 2002; Leon,Cordoba \& Carreño, 2016). El agua Esta se ve afectada principalmente por los vertimientos no controlados provenientes del sector agropecuario, doméstico e industrial (Lizarazo y Orjuela, 2013; Rangel et al., 2019). Más aún, las actividades humanas alteran los procesos evolutivos del paisaje y modelan sus formas naturales; por ende, de la manera como la población se organice en el territorio es uno de los detonantes para los conflictos ambientales (Agualimpia Dualiby \& Castro Méndez, 2012). Por otro lado, Benítez y Contreras (2013) explican que no solo los desechos domésticos afectan las cuencas hídricas, determinan que la agricultura tiene una alta influencia por el uso inadecuado y excesivo de grandes volúmenes de plaguicidas; como resultante genera la contaminación de las aguas superficiales cercanas a las zonas de producción. Los plaguicidas pueden llegar a ser consumidos a través del agua potable, ya que estas sustancias químicas pueden persistir por largos periodos de tiempo en las aguas subterráneas y superficiales (del Puerto Rodríguez, Suárez Tamayo \& Palacio Estrada, 2014). Por lo anterior, debido a la contaminación presente en las fuentes hídricas, el agua sufre cambios en su color y estructura, por la diversidad de compuestos que llegan a ella: desechos de los hogares, detergentes, petróleo, pesticidas y desechos nucleares (Peñaloza Paez, 2012; Vaca, Ubaque \& de Plaza Solórzano, 2016). Además, la dispersión de la contaminación sobrepasa los límites geopolíticos; razón por la cual la calidad del agua en una cuenca es un indicador del impacto de las actividades humanas en una determinada zona (Torres Beristáin et al. 2013). A su vez, Larios Ortiz (2009) enuncia que la cantidad de agua subterránea disponible se ve afectada por el sobreuso de productos agroindustriales ya que estos contienen nitratos altamente contaminantes. Gil et al. (2012) establecen que los contaminantes emergentes (CE) son los compuestos de origen químico que al estar presentes en los cuerpos de agua tienen un alto impacto ambiental, que además se refleja en daños a la salud de las poblaciones aledañas.

En el río Ebro, de España, por la actividad agrícola como la ganadera así como algunas actividades industriales, se identificaron fármacos y algunos plaguicidas; sustancias como acetaminofén, atenolol, carbamazepina, ibuprofeno son vertidos al río y en su gran mayoría no alcanzan a ser diluidos por las plantas de tratamiento (Prados Joya, 2010).

En el río San Lucas, en Perú, se identificó la afectación del afluente por heces humanas y de animales, y la disposición de basura en sus riveras; adicionalmente contiene minerales y compuestos químicos por las deficientes prácticas agrícolas conexas al río (Mendoza Astopilco, 2010).

Por otro lado, en México, el río Atoyac se ve afectado por desechos industriales que han impactado a más de 2300000 habitantes en 28 
municipios. El agua se torna de colores oscuros debido a su nivel de contaminación; y se han encontrado 25 sustancias nocivas que pueden ocasionar enfermedades como hepatitis, cáncer y cólera (Estrada Rivera, 2018).

Hay que mencionar que la materia fecal es una de las principales problemáticas presente en el río Poas, en Costa Rica, en donde el nivel y concentración en temporada seca aumentan, debido a que el agua no tiene flujo constante; además, hay incremento en la propensión y riesgo de contraer enfermedades por esta causa también (Brenes \& Molina, 2012).

Por otro lado, López et al. (2012) muestran que en Colombia el volumen de aguas residuales generadas es aproximadamente un $70 \%-80 \%$ del volumen consumido como potable; "así mismo, cabe señalar que las plantas de tratamiento de estas aguas en el país, solo tratan el $43 \%$ de las aguas de los municipios que han sido recolectadas" (Ramírez Hernández, 2015, pág. 296) mostrando las deficiencias en cobertura del país.

Ilustrando lo anterior "durante muchos años, los ríos y quebradas que cubren el territorio nacional han servido como medio de recepción de aguas negras, domésticas, industriales, agropecuarias y desechos sólidos" (Solano, 2012, pág.10). Se presenta un panorama de impactos negativos, "en Colombia, los problemas ambientales tienen diferentes causas, una de ellas es la ausencia de una cultura ambiental en las comunidades que viven o frecuentan zonas aledañas a las corrientes hídricas" (Plata e Ibarra, 2015, pág. 235). A continuación, se presenta el estado de algunos afluentes de la geografía nacional.

En el caso de la cuenca del río Tunjuelo, por efecto de la expansión urbana de la ciudad de Bogotá, se presenta erosión, empobrecimiento del suelo, contaminación del agua y aire (Pachón y Urrego, 2015).

De igual modo el río Cauca presenta moléculas de plomo, cadmio, cromo y mercurio. Se estima que el departamento de Risaralda produce alrededor de 50000 toneladas de desechos y materiales contaminantes que son vertidos al río (Reyes et al. 2016).

De acuerdo con lo anterior, los impactos generados en el río Magdalena, que oferta el 10,6 \% del recurso hídrico del país, son altos debido a su sobreexplotación y a su aporte en el valor al producto interno bruto (PIB) nacional. A su vez, se presentan casos similares con las vertientes del Orinoco, Amazonas, Sinú, Atrato y Catatumbo, que aún no tienen el nivel de deterioro del Magdalena, pero presentan una alta intervención antrópica (Cepal, 2002).

Por último, se asocia la contaminación de los afluentes con enfermedades en las poblaciones aledañas; principalmente en el sur del departamento de Bolívar, ya que allí se vierten algunos metales pesados y plaguicidas que han generado malformaciones como labio leporino y paladar hendido (Ortega-Miller, 2017).

Por otro lado, el departamento del Meta es un gran productor hídrico ubicado en la región de los Llanos Orientales de Colombia; uno de sus grandes afluentes es el río Meta que tiene una superficie de cuenca de 93800 km² (Junguito Bonnet, 2011) su cauce cruza el municipio de Puerto López en donde se destacan, según el Plan Básico de Ordenamiento Territorial (PBOT), seis subcuencas, que ocupan un área de 166.25 ha y sus playas ocupan 45.06 ha, constituyéndose el rio Metica como el principal drenaje del municipio (se vierte el 67\% de las aguas del alcantarillado), siendo una de estas subcuencas donde se encuentra el caño Banderas, que es el objeto de estudio de este trabajo.

Puerto López se localiza en el nororiente del departamento, es conocido como el centro geográfico de Colombia, debido a que en el alto Menegua se encuentra el centro del país; está ubicado a una distancia de $90 \mathrm{~km}$ de la capital, Villavicencio; limita por el sur, con el municipio de San Martín; por el Oriente, con el municipio de Puerto Gaitán; por el norte, con el departamento de Casanare y el municipio de Cabuyaro; por el occidente, con los municipios de San Carlos y Villavicencio. Puerto López cuenta con una extensión 
de $6290 \mathrm{~km}^{2}$ en la zona rural y $8 \mathrm{~km}^{2}$ en la zona urbana; la topografía es en su mayoría plana y presenta paisajes propios del Ilano (Alcaldía de Puerto López, 2016).

Adicionalmente, el Plan de Desarrollo "Puerto López para todos", del periodo 2016-2019, indica que el municipio está estructurado y dividido en el perímetro urbano con 21 barrios, y el sector rural está conformado por 10 corregimientos de policía y 20 veredas catastrales. En la zona urbana, los barrios Abel Rey, Policarpa y Santander fueron seleccionados como transecto de estudio, dado que estos se encuentran ubicados al margen del afluente.

Por ende, el caño Banderas quien recibe el $33 \%$ de las aguas negras del municipio, pertenece a la cuenca del río Metica. Identificando adicionalmente, el caño de Los Muertos que desemboca al caño Banderas, indicando que se encuentra contaminado por las aguas residuales y basuras que son allí arrojadas. Además, en cuanto al tratamiento de las aguas residuales, el documento emanado por la Corporación para el Desarrollo Sostenible del Área de Manejo Especial La Macarena (Cormacarena, 2014) establece que el municipio debe incluir en el PBOT los sitios de ubicación de las plantas de tratamiento de aguas residuales (PTAR), incluyendo sus respectivas zonas de amortiguación.

En el presente trabajo se identifican los puntos de impacto ambiental del caño Banderas en el transecto seleccionado y se comparan las acciones propuestas en cuanto a saneamiento básico y residuos sólidos en el PBOT y el Plan de Desarrollo de Puerto López 2016-2019 frente a los hallazgos en campo. Por ende, el alcance del trabajo del mismo está dado en términos de establecer la situación actual del caño banderas frente a la gestión técnica y de planeación del municipio.

\section{METODOLOGÍA}

El presente trabajo comprendió tres fases, que incluyeron tareas de revisión documental como también trabajo de campo complementarios: en la primera, se desarrolló la recopilación documental y generación de información base; en la segunda, se llevó a cabo contacto con la comunidad; por último, en la tercera, se realizó el procesamiento e interpretación de la información obtenida en las etapas previas.

Inicialmente, se revisó y analizó la información plasmada en los documentos municipales, como el Plan de Acción de Puerto López y su Plan Básico de Ordenamiento Territorial. Se identificaron las afectaciones, causas, medidas o estrategias implementadas, así como su resultado en términos de solución. Lo anterior con el fin de realizar una comparación con el trabajo de campo para cumplir con el objetivo de este trabajo.

Posteriormente se dio inició a la interacción de contexto en el caño Banderas; para lo que se utilizó un enfoque mixto que permitió tener una percepción adecuada del entorno y sus relaciones (Hamui-Sutton, 2013). Se inició con entrevistas semiestructuradas (Izcara y Andrade, 2013) con el periodista Apolinar Beltrán que trabaja para el periódico regional Tierra Mágica, y con Juan Martínez, presidente de la junta de acción comunal de los barrios localizados en el área de estudio del caño Banderas. Estas dos personas se constituyeron como informantes principales ya que son conocedoras de la zona y han implementado denuncias públicas sobre la contaminación y el riesgo para la comunidad.

En contraste, se realizó una encuesta a las 82 familias que habitan los barrios de la zona, donde se determinó el grado de conocimiento que tiene la población acerca de la afectación de su entorno, cuáles son las causas y de qué manera están implicados.

Para el procesamiento de la parte cuantitativa de la información se utilizó el software SPSS (Castañeda, 2010); a través del cual se identificaron las relaciones entre participación ciudadana, gestión del gobierno, conservación y contaminación.

Se usó también, Atlas.ti para el análisis cualitativo (Taylor \& Bogdan, 1987) de datos, y se analizaron las entrevistas en profundidad; la 
categoría determinada fue conocimiento de la contaminación ambiental, y las subcategorías aplicadas gobierno, comunidad, factores contaminantes, acciones realizadas.

\section{RESULTADOS Y DISCUSIÓN}

En el análisis cuantitativo se estableció la relación entre contaminación y la participación ciudadana, haciendo referencia al impacto que tiene la comunidad en el agua y riberas del caño; se identificó que si existiera participación e interés por parte de los habitantes de la zona se disminuiría el nivel de contaminación del afluente; pero ellos, en un alto porcentaje, depositan residuos sólidos y vertimientos en el cuerpo de agua y no implementan medidas de mitigación del impacto negativo producido por ellos.

Además, al relacionar las variables gestión del gobierno y conservación se identifica que la institucionalidad es deficiente en su intervención frente a la conservación; este resultado se refleja en la ausencia de acciones por parte del gobierno municipal en el cauce del caño a pesar de que está contenido en el PBOT y existen contratos para su mejoramiento.

La comunidad presente en la zona determinó, que el impacto recurrente es por residuos sólidos, con un 49 \% para el subítem de muy alto, motivado por la ausencia de cultura ciudadana, así como educación ambiental. Le sigue un $45 \%$ por aguas servidas, debido a que un $90 \%$ de las viviendas que colindan con el caño no cuentan con servicio de alcantarillado, por lo que se ven obligados a utilizar el caño para suplir este servicio, aunado a que el municipio no cuenta con una PTAR en funcionamiento ni una red de alcantarillado óptima, lo que origina que gran parte de los descoles sigan vertiendo al cuerpo de agua. En un tercer lugar aparece la deforestación con un $41 \%$, ya que en época de sequía la población tala árboles para construir parte de sus casas, corrales o para utilizarlos como leña. Con un $20 \%$ impacta la ganadería, ya que los animales de granja, como gallinas, cerdos, vacas y caballos afectan el agua con sus heces, además generan compactación y sedimentación a lo largo de caño.

Adicionalmente, el $49 \%$ de los encuestados manifestó que siempre observan personas arrojando desechos al caño, mientras que el $46 \%$ determinó que casi siempre y solo un $5 \%$ a veces observa personas arrojando basura.

Para la fase cualitativa, el programa Atlas.ti 7 permitió analizar las entrevistas semiestructuradas; se establecieron cuatro subcategorías y sus descriptores: comunidad, acciones realizadas, gobierno y factores contaminantes. Inicialmente, los informantes destacaron la situación de ocupación informal en la que se encuentran los habitantes de estos barrios, así como las necesidades por las que pasan al no tener los servicios públicos; además, se refleja en sus narraciones la falta de conciencia e interés de la mayoría de la población tanto como la renuencia a mitigar el impacto por ellos generado.

Por otro lado, identificaron entre los contaminantes el muro de contención, construido en la margen izquierda del caño, que impide que tenga un curso normal, apozando el agua y residuos sólidos contaminando el entorno. Otro factor es la ausencia del sistema de alcantarillado en los tres barrios implicados en la problemática, lo que genera que las aguas servidas sean vertidas al caño Banderas.

En el factor gobierno se denota y reitera el incumplimiento por parte de este, ya que algunos proyectos como la reforestación del caño Banderas no fueron realizados; así mismo, se destinaron recursos para la construcción de la PTAR, pero aún no se ejecuta. Por otro lado, se han interpuesto recursos jurídicos a la Alcaldía Municipal, pero estos no han sido resueltos favorablemente.

Por otro lado, el programa arrojó una nube de palabras indicativas de relevancia, en donde caño fue mencionada en más ocasiones, puesto que los entrevistados la utilizaban para referirse a la zona problema. Igualmente, la palabra alcantarillado hacía referencia a su ausencia en los tres barrios 
objeto de estudio, con el agravante de que los desechos sólidos que corren por el drenaje de aguas lluvias del municipio se vierten en el caño.

El término contaminación se utilizó para destacar las causas que afectan este afluente, como lo es el muro de contención, las basuras arrojadas por la comunidad, las cocheras, la falta de conciencia, entre otras. Así mismo, mediante la palabra alcaldía se identificó el desinterés y la negligencia por parte de la institucionalidad, puesto que no se han tomado medidas que mitiguen los impactos negativos presentes. Finalmente, el término problema describió las afectaciones que influyen en la contaminación del caño Banderas.

Por todo lo descrito, el caño Banderas se ve afectado por diversas intervenciones, una de ellas es el muro de contención, el cual separa al caño de su entorno natural, además los residuos sólidos que son arrojadas allí están quedando atrapados constituyendo un cúmulo de desechos contaminantes. Adicionalmente, la falta de conciencia en la comunidad es plausible en cada una de las acciones representadas en los residuos depositados a un costado del muro; el inadecuado manejo administrativo que se le está dando a los planes de gobierno son un detonante, pues su incumplimiento deteriora de la zona; los contratos adjudicados y no ejecutados o realizados en forma parcial redundan en detrimento ambiental.

El Plan Básico de Ordenamiento Territorial contempla la evaluación de la contaminación en el caño Banderas, pero en ningún momento se plantean medidas de solución o estrategias en apoyo con la comunidad.

\section{CONCLUSIONES}

La carencia en la prestación de servicios públicos, por constituirse como un asentamiento informal, es determinante para la generación constante de vertimientos y desechos sólidos; también, la falta de conciencia por parte de la comunidad asentada en la ribera del caño Banderas incrementa los impactos negativos de la zona.
Por otro lado, se determinó la existencia contractual de mejoramientos del transecto estudiado, pero desafortunadamente no fueron ejecutados ni se presentan recursos judiciales al respecto.

Son necesarias sinergias entre la institucionalidad y la comunidad afectada a través de iniciativas sociales enfocadas a la reforestación y recuperación del cuerpo de agua, ya que no se observó intervención alguna por parte de la municipalidad $y$, a su vez, son muy pocas las personas que han creado iniciativas o propuestas ambientales para ser aplicadas en el sector.

Finalmente, se establece que en el caño Banderas no se ha generado un mejoramiento de la sostenibilidad ambiental en los últimos años; por el contrario, se observa un incremento constante en los impactos negativos presentes.

\section{RECOMENDACIONES}

Es necesario plantear alternativas de reforestación con especies nativas a lo largo del cauce para mitigar su estado actual, para lo cual es indispensable conseguir el apoyo económico y adquirir el compromiso con las diferentes entidades públicas y privadas de la región.

Se deben encaminar acciones entorno a la intervención de Cormacarena como la institución enfocada a la preservación del ambiente. A su vez, las empresas de la región, como Riopaila y Bionergy, tienen programas de compensación forestal; al solicitar su apoyo, se podría lograr el mejoramiento de la zona.

Se propone la realización de charlas, talleres y actividades lúdicas con la intervención de la Institución Educativa Técnico Agropecuario, así como con la Universidad de los Llanos, con el fin de crear jornadas de sensibilización y limpieza del caño. Estas actividades son una gran oportunidad para generar compromiso con la comunidad. También, a través del presidente de la Junta de Acción Comunal se puede convocar a las familias que están directamente afectadas para organizar reuniones en las que se establezcan planes de limpieza y el cuidado de la zona. 
En cuanto a las basuras, se deberá solucionar con Espuerto S. A., la cual es la entidad encargada de la recolección de desechos, proponiendo la ubicación de contenedores en puntos clave de los barrios afectados, con el fin de que las personas puedan disponer adecuadamente sus residuos, ya que en la actualidad no lo hace.

En época seca, la Alcaldía deberá imponer sanciones económicas y culturales a quienes talen arboles como una medida correctiva; esto deberá ser tratado en las diferentes actividades que se realicen con la comunidad.

Así mismo, es necesario establecer un programa de saneamiento predial por parte de la Alcaldía o, por el contrario, uno de reubicación para la recuperación de la zona de protección de ronda de caño.

Se deben implementar los respectivos cierres de las porquerizas presentes; así como el aislamiento de la zona de protección de la ocupación de semovientes.

La propuesta de demolición del muro perimetral es pertinente, así como la adecuación del sistema de aguas Iluvias y alcantarillado.

\section{AGRADECIMIENTOS}

A Julián García Sánchez y Brayan Herrera, quienes facilitaron el contacto con las comunidades, así como la recolección de información.

\section{REFERENCIAS}

Agualimpia Dualiby, Y. D. C., \& Castro Méndez, C. E. (2012). Aprovechamiento responsable del recurso hídrico fluvial. Ingeniería Hidráulica y Ambiental, 33(3), 18-32.

Alcaldía de Puerto López, Meta. (2016). Plan básico de ordenamiento territorial municipio de Puerto López diagnostico urbano. Puerto López

Barba Ho, L. E. (2002). Conceptos básicos de la contaminación del agua y parámetros de medición [Universidad del Valle]. https://www.coursehero.com/ file/20925691/conceptos/
Benítez-Díaz, p., \& Miranda-Contreras, L. (2013). Contaminación de aguas superficiales por residuos de plaguicidas en Venezuela y otros países de Latinoamérica. Revista Internacional de Contaminación Ambiental, 29, 7-23.

Brenes, G. C., \& Molina, J. M. (2012). Contaminación fecal en varios ríos de la Gran Área Metropolitana y la Península de Osa. Tecnología en Marcha, 25(4), 33-39. 10.18845/tm.v25i4.617

Castañeda, M. B. (2010). Procesamiento de datos y análisis estadísticos utilizando SPSS: Un libro práctico para investigadores y administradores educativos. Edipucrs.

Cepal. (2002). La contaminación de los ríos y sus efectos en las áreas costeras y el mar (50). Recuperado de https://archivo.cepal.org/pdfs/Waterguide/ LCL1799S.pdf

Cormacarena, Corporación para el Desarrollo Sostenible del Área de Manejo Especial La Macarena. (2014), Términos De Referencia Para La Formulación De Planes De Saneamiento Y Manejo De Vertimientos (Psmv), Grupo Agua Subdirección De Gestión Y Control Ambiental.

del Puerto Rodríguez, A. M., Suárez Tamayo, S., \& Palacio Estrada, D. E. (2014). Efectos de los plaguicidas sobre el ambiente y la salud. Revista Cubana de $\mathrm{Hi}$ giene y Epidemiología, 52(3), 372-387.

Estrada Rivera, A. (2018). Evaluación toxicológica del agua residual textil (proceso DEMIN) vertida al río Atoyac (Master's thesis), Benemérita Universidad Autónoma de Puebla.

Gil, M. J., Soto, A. M., Usma, J. I., \& Gutiérrez, O. D. (2012). Contaminantes emergentes en aguas, efectos y posibles tratamientos. Producción+ limpia, 7(2).

Hamui-Sutton, A. (2013). Un acercamiento a los métodos mixtos de investigación en educación. Investigación en Educación Médica, 2(8), 211-216. 10.1016/S2007-5057(13)72714-5

Izcara, S. y Andrade, K. L. (2013). La entrevista en profundidad teoría y práctica. Tamaulipas: Universidad Autónoma de Tamaulipas (UAT).

Junguito Bonnet, R. (2011). Transportes fluviales y desarrollo empresarial en Colombia: la empresa El 
Libertador de Navegación a Vapor por el Río Meta, 1892-99 (Doctoral dissertation, Facultad de Ciencias Económicas. Universidad de Buenos Aires).

Larios Ortiz, L. (2009). Metahemoglobinemia en niños: situación Actual. Revista Archivo Médico de Camagüey, 13(3).

León Agatón, A., Córdoba Ruiz, J. C., \& Carreño Sayago, U. F. (2016). Revisión del estado de arte en captación y aprovechamiento de aguas Iluvias en zonas urbanas y aeropuertos. Tecnura, 20(50), 141-153.

Lizarazo Becerra, J. M., \& Orjuela Gutiérrez, M. I. (2013). Sistemas de plantas de tratamiento de aguas residuales en Colombia (Doctoral dissertation, Universidad Nacional de Colombia).

López, J. R., Elías, A., Delgado, D., Sarduy, L., \& Domínguez, M. (2012). Efecto de la suplementación con concentrado proteico energético en la dinámica de las partículas del rumen en bucerros (Bubalus bubalis) alimentados con pasto estrella (Cynodon nlemfuensis). Livestock Res. Rural Devel, 24, 158.

Mendoza Astopilco, V. E. (2010). Estudio de la contaminación del río San Lucas (Doctoral dissertation, Tesis, Municipalidad Provincial de Cajamarca, Perú).

Ortega-Miller, J. G., Yezioro-Rubinsky, S., Benavides-Pinto, B. C., \& Báez-Quintero, L. C. (2017). Efectos teratogénicos de insecticidas organofosforados en la etiología de labio y paladar hendido: revisión de literatura. Revista Nacional de Odontología, 13(24). 10.16925/od.v12i24.1658

Pachón, M. J. C., \& Muñoz, L. M. U. (2016). Problemáticas socioambientales en la cuenca del río Tunjuelo: una posibilidad en la enseñanza y el aprendizaje de la geografía. Revista de Geografia do Colégio Pedro II, 2(4), 95-108

Peñaloza Paez, J. A. (2012). LA CONTAMINACIÓN AMBIENTAL. DELOS Desarrollo Local Sostenible, 5(N13), 1-5. https://www.eumed.net/rev/delos/13/ japp.html

Plata, Á. M., \& Ibarra Vega, D. (2016). Percepción local del estado ambiental en la cuenca baja del río Manzanares. Luna Azul, (42), 235-255. 10.17151/ luaz.2016.42.15
Prados Joya, G. (2010). Tratamiento de aguas para la eliminación de antibióticos -nitroimidazoles- mediante adsorción sobre carbón activado y tecnologías avanzadas de oxidación. http://hdl.handle. net/10481/49621

Rangel, B. C. S., Olveira, A. E. M., Arellano, V. H. R., \& González, C. A. G. (2019). Nanomateriales celulósicos para la adsorción de contaminantes emergentes. Tecnura, 23(62).

Ramírez Hernández, O. (2015). Identificación de problemáticas ambientales en Colombia a partir de la percepción social de estudiantes universitarios localizados en diferentes zonas del país. Revista internacional de contaminación ambiental, 31(3), 293-310 10.20937/rica.53548

Reyes, Y., Vergara, I., Torres, O., Lagos, M. D., \& Jimenez, E. E. G. (2016). Contaminación por metales pesados: Implicaciones en salud, ambiente y seguridad alimentaria. Ingeniería Investigación y Desarrollo: $12+D, 16(2)$, 66-77. 10.19053/1900771X. v16.n2.2016.5447

Solano, M. (2011). Impacto ambiental por aguas residuales y residuos sólidos en la calidad del agua de la parte media-alta de la microcuenca del río Damas y propuesta de manejo. Tesis, Universidad Nacional de Costa Rica, Heredia, Costa Rica.

Taylor, S. J., \& Bogdan, R. (1987). Introducción a los métodos cualitativos de investigación (Vol. 1). Barcelona: Paidós.

Torres Beristáin, B., González López, G., Rustrián Portilla, E., \& Houbron, E. (2013). Enfoque de cuenca para la identificación de fuentes de contaminación y evaluación de la calidad de un río, Veracruz, México. Revista internacional de contaminación ambiental, 29(3), 135-146.

Vaca, M. C. G., Ubaque, C. A. G., \& de Plaza Solórzano, J. S. (2016). Estudio exploratorio del tratamiento de agua de lavado de tintas por método de electrocoagulación/electroflotación. Tecnura, 20(47), 107117. 10.14483/udistrital.jour.tecnura.2016.1.a09

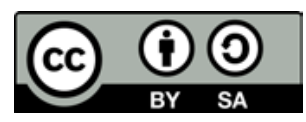

Tecnura • p-ISSN: 0123-921X • e-ISSN: 2248-7638 • Vol. 24 No. $65 \bullet$ Julio - Septiembre de $2020 \bullet$ pp. 77-84 